\title{
Circuit
}

Musiques contemporaines

\section{Voyage au bout de l'oeuvre : Prologue au numéro}

\section{Jonathan Goldman}

Volume 18, numéro 3, 2008

Claude Vivier, vingt-cinq ans après : une introspection

URI : https://id.erudit.org/iderudit/019136ar

DOI : https://doi.org/10.7202/019136ar

Aller au sommaire du numéro

Éditeur(s)

Les Presses de l'Université de Montréal

ISSN

1183-1693 (imprimé)

1488-9692 (numérique)

Découvrir la revue

Citer ce document

Goldman, J. (2008). Voyage au bout de l'oeuvre : Prologue au numéro. Circuit, 18(3), 5-7. https://doi.org/10.7202/019136ar d'utilisation que vous pouvez consulter en ligne.

https://apropos.erudit.org/fr/usagers/politique-dutilisation/ 


\section{Voyage au bout de l'œuvre: Prologue au numéro}

Jonathan Goldman

Vivier. Claude Vivier. Comme on dit Mozart. Wolfgang Amadeus Mozart... N'est-il pas normal de reconnaître nos propres héros? Si la musique est une expression fondamentale de l'Humanité, alors Claude Vivier a su exprimer la quintessence de notre culture, de notre histoire, de nos rêves. Vivier, c'est un véritable «trésor national»!

Walter Boudreau (dans le communiqué de presse de la série Hommage à Claude Vivier de la SMCQ)

Ce n'est certainement pas diminuer la portée des réalisations de Claude Vivier (1948-1983) que de relever que nous assistons à la construction d'un mythe autour du grand compositeur québécois, qualifié de «héros» et de «trésor national» par Walter Boudreau - et, à travers sa voix, par de nombreux musiciens d'aujourd'hui. Cette consécration a lieu au moment du $60^{\mathrm{e}}$ anniversaire de la naissance et, triste calcul, du $25^{\mathrm{e}}$ anniversaire de la mort de Vivier. Aussi devons-nous analyser sur quoi repose le mythe Vivier, non pas pour le récuser ou le déconstruire, mais simplement pour comprendre ce que ce phénomène nous dit de notre société, de qui nous sommes.

Il ne saurait s'expliquer entièrement par la reconnaissance dont l'œuvre de Vivier a joui à l'étranger (dont les trois manifestations les plus importantes sont: le jugement maintes fois cité de György Ligeti, qui ferait de Vivier «le plus grand compositeur français [sic] de sa génération »; l'enregistrement de quelques-unes de ses œuvres par un chef de prestige, Reinbert de Leeuw ${ }^{1}$; et l'édition de ses œuvres chez Boosey and Hawkes²); même si ce sont là des facteurs non négligeables, la gloire à l'étranger étant un préalable à la reconnaissance locale dans des sociétés qui manquent de confiance en soi, comme c'est parfois le cas au Québec et au Canada. Nous devons plus généralement nous demander «comment des faits s'inscrivent dans une rhétorique pour orienter la lecture d'une œuvre »3. Après tout, l'« effet» Vivier constitue un phénomène sans précédent dans l'histoire de la musique d'ici, qui mènera vraisemblablement le compositeur au panthéon québécois déjà habité par

1. Le CD EMI Booooo41EX ainsi qu'un DVD, Opus Arte Media BoooCIWXSW.

2. La célèbre maison d'édition de Bartók et de Stravinsky s'est engagée en 2005 à publier la quasi-totalité des œuvres de Vivier; < http ://www.boosey. com/downloads/BH\%20Vivier\%20 signing1.pdf $>$.

3. Benoît Melançon, "VLB personnage et institution (notes)", Études françaises, vol. $19, n^{\circ} 1,1983$, p. 6 . 
4. Benoît Melançon, Les yeux de Maurice Richard. Une histoire culturelle, Montréal, Fides, 2008.
5. Jacques Marchand, Claude Gauvreau, poète et mythocrate, Montréal, VLB éditeur, 1979, p. 11; cité in Melançon, 1983, op. cit., p. 6. des écrivains comme Émile Nelligan, Réjean Ducharme, Michel Tremblay, Victor-Lévy Beaulieu, Saint-Denys Garneau ou Claude Gauvreau, des plasticiens comme Borduas, Riopelle ou Molinari, ou - pourquoi pas? - un athlète comme Maurice Richard ${ }^{4}$; ce serait d'autant plus salutaire que, comme on le note souvent, aucun compositeur québécois n'avait jusqu'ici atteint ce statut au sein de l'imaginaire collectif. Ce phénomène de canonisation (dans le double sens - double seulement en apparence - d'une béatification, le fait de devenir un saint, et de l'intégration de ses œuvres dans un répertoire transhistorique de chefs-d'œuvre absolus) doit être compris grâce à tous les outils mis à la disposition du chercheur par l'analyse musicale, la sociologie de la musique et l'esthétique de la réception.

Certains éléments de ce mécanisme de construction du mythe sont néanmoins évidents, et relèvent d'un effort conscient et collectif de la part des principaux acteurs du milieu québécois de la musique contemporaine : grâce à la SMCQ et à l'initiative de son directeur artistique Walter Boudreau et de sa directrice générale Pierrette Gingras, 2008 a été transformée en véritable année Vivier : la SMCQ lance avec une programmation axée sur l'œuvre de Vivier sa série SMCQ Hommage, qui consacre tous les deux ans une série de concerts à un seul compositeur. Au cours de cet événement, une vingtaine de programmes sont proposés, et autant d'œuvres de Vivier présentées, dont certaines quasi inédites (Learning [1976], partiellement reprise dans Et je reverrai cette ville étrange [1981]), ainsi que plusieurs arrangements (parfois deux le même soir!) de sa célèbre œuvre pour instrumentation libre, Pulau Dewata, dont certains seront examinés dans l'article de Fabrice Marandola dans ce numéro. Si la SMCQ chapeaute et produit l'événement, elle a sollicité la participation de bien d'autres ensembles et organismes, dont la Schulich School of Music de l'Université McGill, le festival Orgue et couleurs, le Quatuor Bozzini, le Studio de musique ancienne de Montréal (SMAM), la Nef, Constantinople, Arraymusic (de Toronto), etc. Dans le volet «Vivier jeunesse", la contribution de la SMCQ Jeunesse à cette année Vivier, la dessinatrice et compositrice Sylvie-Anne Ménard (dite Zviane) propose un album de bandes dessinées dont le héros est Claude (Des étoiles dans les oreilles), qui a été lu par quelque 13 ooo écoliers.

Dans son étude sur la construction du mythe de l'écrivain Victor-Lévy Beaulieu, Benoît Melançon cite Jacques Marchand, qui, en étudiant la construction du mythe de Claude Gauvreau, parle d'«une fascination hypnotique pour le personnage» qui permet d'entretenir «un silence unanime, révérencieux, face à l'œuvre elle-même5.» C'est précisément pour éviter ce travers fréquent de la mythification - le renoncement à interroger l'œuvre - 
qu'est né le projet de ce numéro axé sur l'analyse, dirigé par le compositeur (et membre du comité de rédaction de Circuit) Jean Lesage. Il y a 17 ans déjà, en 1991, Circuit a consacré un double numéro aux écrits de Claude Vivier (vol. 2, nº 1-2); dans le présent numéro, en nous attaquant à l'œuvre musical à proprement parler, nous chercherons à ouvrir des perspectives d'approche de cet œuvre riche et multiforme, dont on signale souvent le caractère hautement personnel, pour ne pas dire «autobiographique », du langage (trop rare en matière de musique contemporaine?). La SMCQ est notre partenaire à part entière dans cet exercice de réflexion, comme en témoigne le Concours international de rédaction d'un texte sur Claude Vivier, lancé par la SMCQ conjointement avec notre revue, et jugé par un jury constitué de membres de Circuit et de la SMCQ, ainsi que d'un musicologue externe. L'essai du lauréat de ce concours, Stephen Rogers, qui explore de façon fort originale le thème du voyage dans l'œuvre de Vivier, est publié dans ce numéro, et son auteur recevra un prix de $1000 \$$. Nous remercions chaleureusement toute l'équipe de la SMCQ pour son aide et son soutien. Et nous avons d'ores et déjà le plaisir d'annoncer qu'un concours semblable sera organisé à l'occasion de la prochaine série Hommage, en 2010, consacrée à un héros bien vivant cette fois: Gilles Tremblay. Comme pour la présente édition, le texte du lauréat sera publié dans Circuit.

En ce qui concerne les autres contributions de ce numéro, alors que Louise Bail, partant de Kopernikus, interroge le thème du sacré chez Vivier, Martine Rhéaume étudie les profils mélodiques employés dans quatre œuvres vocales, découvrant, à partir d'une méthode paradigmatique, un haut niveau de stabilité dans l'écriture. Se penchant sur le matériau mélodique et harmonique dans le style tardif de Vivier, Patrick Levesque analyse quant à lui un langage que l'on peut qualifier de spectral, si du moins l'on admet que le projet musical de ramener l'élaboration harmonique à la composition du timbre aura été partagé, dans le dernier quart du xx siècle, par beaucoup plus de compositeurs que ceux habituellement assimilés à l'ensemble l'Itinéraire ${ }^{6}$. Enfin, dans le Cahier d'analyse, Jean Lesage replace Siddhartha dans le contexte culturel de la classe de Stockhausen et de la Neue Einfachheit.

Il me reste à remercier chaleureusement Jean Lesage, rédacteur invité de ce numéro, qui a eu l'idée de ce projet et qui nous a apporté une aide précieuse dans sa réalisation.

Bonne lecture!
6. Je dois cette observation pertinente à Sean Ferguson lors d'un entretien privé. 